\title{
Femtosecond laser nanostructuring of metal and semiconductor surfaces (Conference Presentation) (Withdrawal Notice)
}

Camilo Florian Baron, Jörg Krüger, Jörn Bonse

Camilo Florian Baron, Jörg Krüger, Jörn Bonse, "Femtosecond laser nanostructuring of metal and semiconductor surfaces (Conference Presentation) (Withdrawal Notice)," Proc. SPIE 11269, Synthesis and Photonics of Nanoscale Materials XVII, 1126908 (27 March 2020); doi: $10.1117 / 12.2542309$

SPIE. Event: SPIE LASE, 2020, San Francisco, California, United States 


\section{Femtosec ond laser nanostructuring of metal and semic onductor surfaces (Conference Presentation) (Withdrawal Notice) Camilo Forian Baron, J örg Krüger, J öm Bonse \\ Bundesanstalt für Ma teria lforsc hung und -prüfung (Gemany)}

Proceedings Volume 11269, Synthesis and Photonics of Nanoscale Materials XVII; 1126908

(2020) https://doi.org/ 10.1117/12.2542309

Event Event: SPIE LASE, 2020, San Francisc 0, Califomia, United States

Online Publication Date: 9 March 2020

Withdrawn from Publication: 25 March 2020

Publisher's Note: This Conference Presentation, orig ina lly published on 9 March 2020, was withdrawn per author request. 